Doi: $10.4274 / j u s .2017 .04 .031$

\title{
Re: Application of Virtual, Augmented, and Mixed Reality to Urology
}

\author{
Hamacher A1, Kim SJ2, Cho ST3, Pardeshi S4, Lee SH4, Eun SJ1, Whangbo TK1 \\ ${ }^{1}$ Gachon University, Department of Computer Science, Seongnam, Korea \\ 2The Catholic University of Korea, Seoul St. Mary's Hospital, College of Medicine, Department of Urology, Seoul, Korea \\ ${ }^{3}$ Hallym University College of Medicine, Department of Urology, Seoul, Korea \\ ${ }^{4}$ Kwangwoon University, Graduate School of Information Contents, Seoul, Korea \\ Int Neurourol J 2016;20:172-181.
}

\section{EDITORIAL COMMENT}

In addition to virtual reality (VR), new developments in augmented reality (AR) (i.e., google glass), and mixed reality (MR) have been introduced in the field of medicine. This review presents an overview of significant developments in VR, AR, and MR technologies which are currently in use in urology, and the future development trends that could be expected. This spectrum depicts a continuum from the real world to the virtual world, leaving space in between for AR as well as for augmented virtuality and considering everything between these two worlds the MR. Usage of $A R, V R$, and MR in medicine is especially focused on neuroscience/psychotherapy, hepatology and orthopedics. However, recent papers reporting 3D image-guided surgery show that AR with visual cues to the subsurface anatomy could be a substitute in minimally invasive surgery in urology. These developments are used for education, 3D modeling of medical imaging visualizations, training, planning, therapy, assisting, telesurgery, telementoring, and augmented biofeedback in pelvic floor muscle re-education. AR and VR have the potential to reduce risk through improved planning and relying on their assistance would reduce the time spent in the operating room. In the near future, these techniques will provide the potential to further increase efficiency in the urological usage.

Fehmi Narter, MD, PhD

${ }^{\oplus}$ Copyright 2017 by the Association of Urological Surgery / Journal of Urological Surgery published by Galenos Publishing House. 